\title{
Fine Sieving of Atmospheric Particles in a Collected Air Sample Using Oil Electrophoresis
}

\section{Aerosol and Air Quality Research}

\section{OPEN ACCESS}

Received: December 22, 2020

Revised: February 15, 2021

Accepted: March 14, 2021

\section{${ }^{*}$ Corresponding Author: yao@pku.edu.cn \\ ${ }^{+}$These authors contributed equally to this work}

\section{Publisher:}

Taiwan Association for Aerosol Research

ISSN: $1680-8584$ print ISSN: 2071-1409 online

\section{Copyright: The Author(s)} This is an open access article distributed under the terms of the Creative Commons Attribution License (CC BY 4.0), which permits unrestricted use, distribution, and reproduction in any medium, provided the original author and source are cited.

\author{
Xinyue $\mathrm{Li}^{\dagger}$, Siyu $\mathrm{Xu}^{\dagger}$, Maosheng $\mathrm{Yao}^{*}$ \\ State Key Joint Laboratory of Environmental Simulation and Pollution Control, College of \\ Environmental Sciences and Engineering, Peking University, Beijing 100871, China
}

\begin{abstract}
To solve the challenge of extracting nano- to micrometer-sized atmospheric particles from a mixed sample, we developed an electrostatic sieve system, the Fine Sieving of Collected Atmospheric Particles using Oil Electrophoresis (iSCAPE), based on the application of an electrostatic field to a non-conductive mineral oil. Using atmospheric samples, which were collected from different cities, in addition to soil and road dust samples, we tested this system under different conditions and found that the "iSCAPE' $d$ " particles moved rapidly at varying velocities and in two opposite directions. The diverse origins of the sample-ambient air, soil, or road dust-exhibited specific charged properties, and clearly affected the electrical mobility, as demonstrated by the graphs, of the particles following the "iSCAPEing," which lasted from seconds to minutes. We also observed an increased abundance of particles in specific mobility ranges. Furthermore, according to our adenosine triphosphate (ATP) monitoring results, the iSCAPE is capable of separating bacterial particles by size and electrical mobility. The experimental data suggests that the iSCAPE relies heavily on the electrostatic field strength, mineral oil viscosity, and run time. In theory, this method can extract any targets from a complex sample, thus creating many research opportunities in environmental, biomedical, and life sciences.
\end{abstract}

Keywords: Atmospheric particles, Sieving, Electrical mobility, iSCAPE, Size distribution

\section{INTRODUCTION}

Air pollution, especially particulate matter (PM), has become one of the most important environmental problems in the world. Exposure to PM has resulted in millions of deaths globally (Forouzanfar et al., 2013). The components of atmospheric particles are very complex, including biologicals such as bacteria, fungi, viruses, pollen and chemical components-sulfate, nitrate, ammonium and other non-biological particles (Zhang et al., 2019). There are some commercially available instruments for studying differently sized atmospheric particles (Agranovski et al., 2003; Yao and Mainelis, 2006a), but some of them do not automatically provide samples for post-analysis. In addition, collection of nanoscale particles requires expensive equipment and high power source (Yue et al., 2018). Differential mobility analyzer (DMA) with up to 192 size channels is otherwise used to study size distributions of nanoscale particles ( $1 \mathrm{~nm}-1 \mu \mathrm{m}$ ) (Hewitt, 1957; Knutson and Whitby, 1975). However, use of such an equipment is often restrictive due to its availability and post-analysis difficulties. For studying PM health effects, it is also challenging to differentiate the toxicity between differently sized particles since they are often mixed together in a sample. Separation and classification of atmospheric particles using currently available methods are often prohibitive in terms with their sizes and species, especially for post-analysis, and equipment cost.

On the other hand, biological detection of certain microbial species is often prohibitive due to complex environmental matrix of the samples, e.g., polymerase chain reaction (PCR) inhibition problems encountered in many studies (Alvarez et al., 1995; Hospodsky et al., 2010; Xu and Yao, 2013). Accordingly, extraction of target agent is desired. In the field of microbiology, the method of gel electrophoresis has been extensively used in separating the DNAs since its earlier invention 
(Panyim and Roger, 1969; Ünlü et al., 1997). On another front, it was revealed that particles in the atmosphere likewise carry different polarity charges and levels (Yao and Mainelis, 2006b; Wei et al., 2014). For example, it was shown that bacterial particles in indoor and outdoor air carried about 21-92 elemental unit charges (Xie et al., 2011). Here, we used the same electrophoresis concept, but applied it to separate particles in a particle mixture collected from ambient air according to their electrical mobility. Particularly, we invented a particle mixture sieving system named iSCAPE by employing an electrostatic field in a non-conductive mineral oil medium. Under the same operating conditions, particles in a sample with different electrical mobility would move at varying velocities in the mineral oil, thus ending up at different locations on the particle moving line within a given time. Depending on objectives, targeted particles or molecules can be thus efficiently extracted from a complex particle of environmental or medical origin using the iSCAPE developed.

\section{MATERIALS AND METHODS}

\subsection{Experimental Setup}

In this work, we pioneered a system named iSCAPE (Fine Sieving of Collected Atmospheric Particles using Oil Electrophoresis) by using an electrical field in a non-conductive liquid (mineral oil) as shown in Fig. 1. The iSCAPE sieves particles of different sizes out from collected atmospheric particle mixture based on their electrical mobility differences. The system consists of four major components: high voltage supply (Model 205B-20R; Bertan, Hicksville, New York, USA), two copper electrodes, mineral oil (M5904; Sigma-Aldrich, USA), and the electrophoresis container (electrical insulation support). In addition, the iSCAPE system is also provided with a ruler that is used to measure the distance from the PM feed point (the middle point of the separation line) as illustrated in Fig. 1. The dimensions of the container are $60 \times 20 \times 4 \mathrm{~mm}$ (length $\times$ width $\times$ height). The power supply can provide a voltage of up to $20 \mathrm{kV}$. The used mineral oil has a viscosity of 14.2$17.2 \mathrm{cSt}(11.9-14.5 \mathrm{mPa} \cdot \mathrm{s})$ and density of $0.84 \mathrm{~g} \mathrm{~mL}^{-1}$ at $25^{\circ} \mathrm{C}$. Different viscosity of mineral oil can be used to modify the particle electrical mobility. The mineral oil is non-conductive medium, and able to maintain the viability of microorganisms collected into it from ambient air (Lin et al., 1999). Without interferences, mineral oil was also used in a PCR reactionto form a vapor barrier to prevent the evaporation of the reaction at elevated temperatures (Sparkman, 1992).

\section{2 iSCAPE of Atmospheric Particles of Various Sizes}

To test the iSCAPE system, we used atmospheric samples previously collected using automobile air conditioning filters for Beijing, Zurich, and San Francisco (Li et al., 2018). Here, we also collected Beijing's soil and road dust samples for testing the system. When operating the iSCAPE, approximately $1 \mathrm{~mL}$ mineral oil was first added into the electrophoresis container. Secondly, the power supply with desired voltage was turned on until being stable without air breakdown between the two electrodes. Lastly, approximately $20 \mu \mathrm{L}$ mineral oil suspension with the tested samples dissolved (atmospheric particulate matter, soil sample or road dusts) was pipetted into

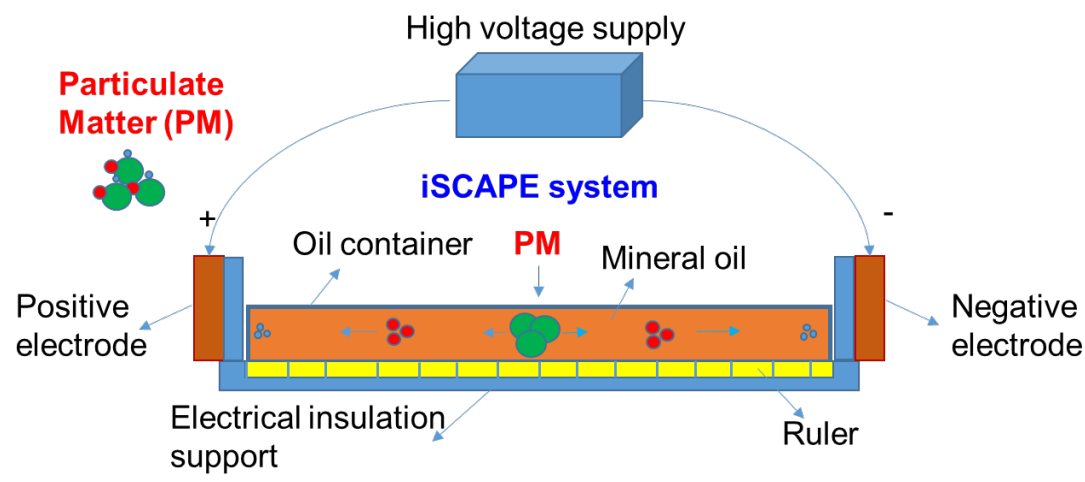

Fig. 1. Experimental setup for fine sieving of atmospheric particles using mineral oil electrophoresis powered by a high voltage supply (iSCAPE). The dimensions of the container are $60 \times 20 \times 4 \mathrm{~mm}$ (length $\times$ width $\times$ height). 
the oil container from the sample feeding point as illustrated in Fig. 1. Depending on the experimental objectives, the tests could last from seconds to minutes to sieve particles or extract desired size particles from the sample mixture. Under the used experimental conditions, particles with different electrical mobility would travel at a different velocity in the mineral oil, thus ending up in different locations away from the sample feeding point. For particles with positive charges, they moved toward the negative electrode, while particles with negative charges moved toward the opposite. The separated samples can be further centrifuged, if needed, to separate the particles from the mineral oil for downstream analysis.

\subsection{Analysis of iSCAPE'd Samples}

In this work, for different samples, we took samples from various points away from the sample feed points, e.g., $0.5,1,1.5,2$ and $3 \mathrm{~cm}$. The samples were further subjected to microscopic analysis using a microscope (BX63; Olympus Corp., Tokyo, Japan). In addition, using a slightly modified iSCAPE system, the particle electrophoresis was also directly conducted on a microscopic slide (S2112; Matsunami Co., Osaka, Japan) such that particles at different points between the electrodes can be continuously imaged using the microscope (corresponding videos are provided in Supporting Information; use of the microscopic slide however could impact the original particle charge distribution in the sample). For particles retrieved from different points between two electrodes, various analyses were conducted. Here, as an example analysis we have calculated their electrical mobility, performed microscopic imaging, and bacterial ATP measurements. The particle electrical mobility was calculated using the following equation (Hinds, 1999):

$\mu_{d}=V_{d} / E=C_{c} \times Q /(3 \times \pi \times d \times \eta)$

where $\mu_{d}$ is the particle electrical mobility $\left(\mathrm{m}^{2} \mathrm{~V}^{-1} \mathrm{~s}^{-1}\right), V_{d}\left(\mathrm{~m} \mathrm{~s}^{-1}\right)$ is the particle velocity, $E\left(\mathrm{~V} \mathrm{~m}^{-1}\right)$ is the uniform electrostatic field, $C_{c}$ is the Cunningham correction factor, $Q$ is the particle charge, $d$ is the particle diameter, and $\eta$ is the medium viscosity. Bacterial ATP measurements, as an example analysis for bacterial separation, were performed using a device (SystemSURE Plus; Hygiena, Camarillo, CA, USA). According to the manufacturer, the device has outstanding performances in terms with its linearity (femtomole ATP vs. log RLU), repeatability ( 5\%), and sensitivity ( 1 femtomole ATP). For measuring their ATP levels, $5 \mu \mathrm{L}$ of mineral oil sample retrieved from different locations was taken and analyzed using the device coupled with a cotton swab.

\subsection{Statistical Analysis}

We have tested the iSCAPE system using different samples (atmospheric PM, soil and road dust samples) under different experimental conditions (different electrostatic field strength (3.17 and $6.33 \mathrm{kV} \mathrm{cm}^{-1}$ ), different run time (20 s to $6 \mathrm{~min}$ ). For each sample retrieved, at least five images were taken from different microscopic views. In addition, we have provided videos of imaged particles along the particle moving lines of the mineral oil. Here, mineral oil (microbiology grade) was also imaged to eliminate the possible particle contamination before any experiments as shown in Fig. $\mathrm{S} 1$.

\section{RESULTS AND DISCUSSION}

\subsection{Sieving of Atmospheric Particles Collected into a Sample by the iSCAPE under Different Electrostatic Field Strength and Run Time}

As shown in Fig. 2, for particles from different cities the iSCAPE has demonstrated different sieving capabilities. For atmospheric samples from Beijing, observed particles seemed to have higher electrical mobility (3.95) compared to those of the particles from San Francisco and Zurich (1.32). In contrast with the control without the iSCAPE, a large amount of particles diffused to $0.5 \mathrm{~cm}$ location from the particle feeding point at a speed of $125 \mu \mathrm{m} \mathrm{s}^{-1}$ under the experimental conditions tested. As observed in Fig. 2, particles with smaller sizes generally moved faster than those larger particles; nonetheless the mobility was proportional to the ratio of particle charge over diameter. Particles from different sources had different particle mobility graphs as shown 

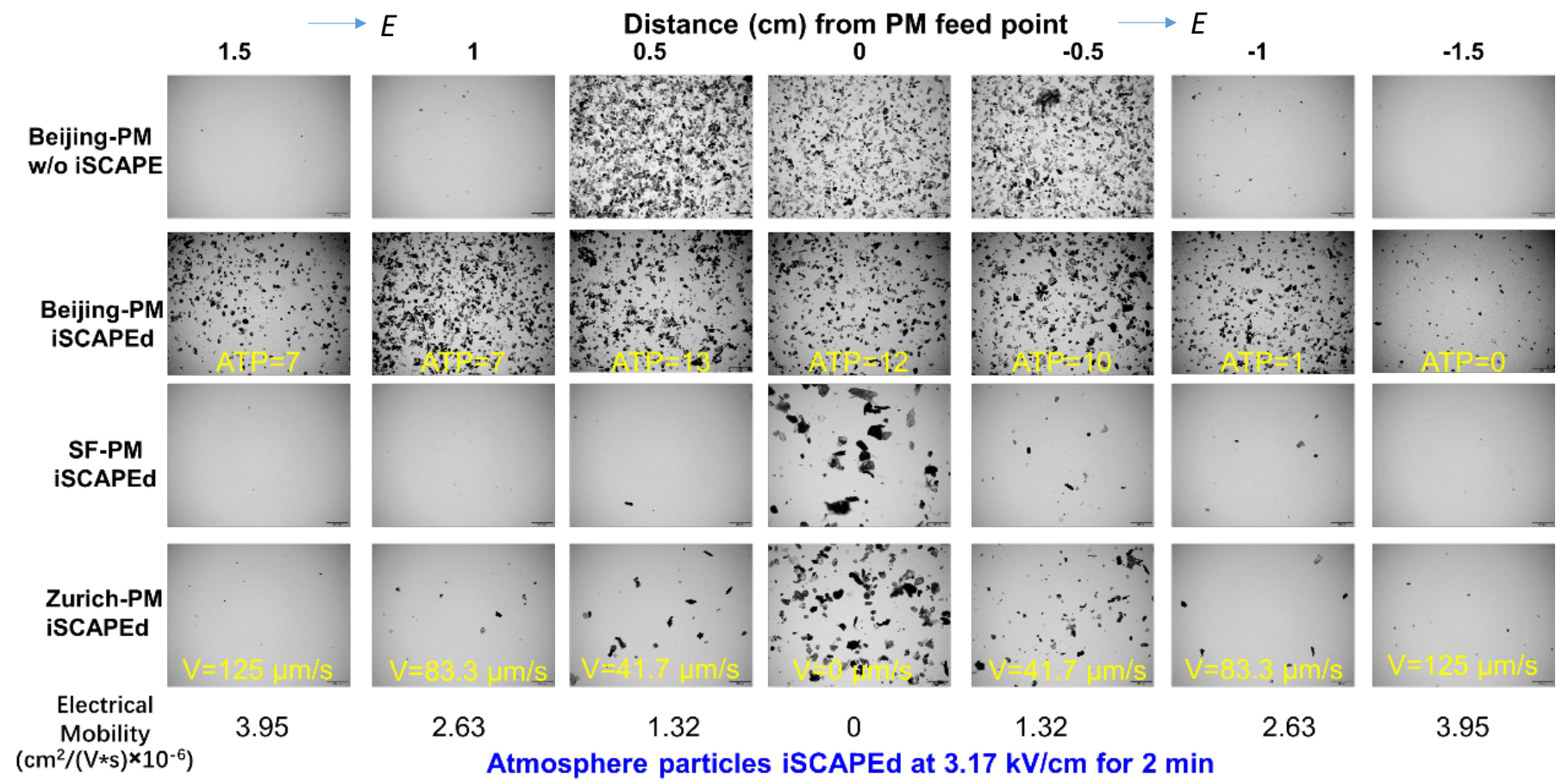

2.63

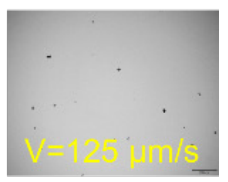

3.95

Fig. 2. Fine sieving of atmospheric particles collected from three global cities (Beijing, Zurich, and San Francisco (SF)) using the iSCAPE at $3.17 \mathrm{kV} \mathrm{cm}^{-1}$ for 2 min. Bacterial ATP results for different sampling points are shown in Fig. 3. For each sampling point, at least five images (200 $\mu \mathrm{m}$ scale bar) taken from different microscopic views of $10 \mu \mathrm{L}$ sample.

in the figure under the same iSCAPE operating conditions ( $3.17 \mathrm{kV} \mathrm{cm}^{-1}$ for $2 \mathrm{~min}$ ). The differences observed for different cities via the iSCAPE were likely due to the different components such as bacteria and metals, and the size distributions of their PM (Li et al., 2018; Yue et al., 2018; Li et al., 2019). For example, air samples from Zurich were shown to have higher fraction of nanoscale particles than those from Beijing (Yue et al., 2018). For the sampling points listed above, Fig. 3 showed the ATP measurements for Beijing's samples that were iSCAPE'd. As seen in the figure, most of bacteria moved to the positive electrode (56\%), concentrating within $1.5 \mathrm{~cm}$ range from the particle feed point (B-0). For the negative electrode, about $21 \%$ was located within $1 \mathrm{~cm}$ range from the feed point. These data suggest that the iSCAPE system can be also used to separate bacterial particles, and a higher fraction of them were shown carrying negative charges. Apparently, a stronger electrostatic field or longer run time are needed to sieve large particles from Zurich and San Francisco. For the ATP data, only one measurement was taken to avoid mineral oil movement and corresponding sample displacement when more volumes of samples were taken from the separation line.

To further test the iSCAPE capability, we have repeated the test with Beijing's PM samples but with longer run time, i.e., $6 \mathrm{~min}$, at the same electrostatic field strength $\left(3.17 \mathrm{kV} \mathrm{cm}^{-1}\right)$ (Fig. 4). Compared to shorter time shown in Fig. 2, more particles travelled away from the PM feed point. It can be again seen that in general particles with smaller sizes travelled much faster $\left(55.6 \mu \mathrm{m} \mathrm{s}^{-1}\right)$ than those with larger sizes $\left(13.9 \mu \mathrm{m} \mathrm{s}^{-1}\right)$. In addition to these sampling points, we have provided particle separation information (iSCAPE'd for $3 \mathrm{~min}$ ) along the particle moving line in videos (File S1 and S2) from which imaged particles can be seen at any locations between the electrodes. Also, as observed from the figure there were more particles carrying negative charges than those carrying positive ones. Data in these videos also demonstrated that the iSCAPE system can efficiently sieve out the particles. Depending on the targets to be obtained, the run time and electrostatic field strength can be fine-adjusted in addition to the mineral oil viscosity.

\subsection{Soil and Road Dust Sample Sieving by the iSCAPE under Different Electrostatic Field Strength and Run Time}

To further validate the iSCAPE system, we have also performed the same tests with Beijing soil 


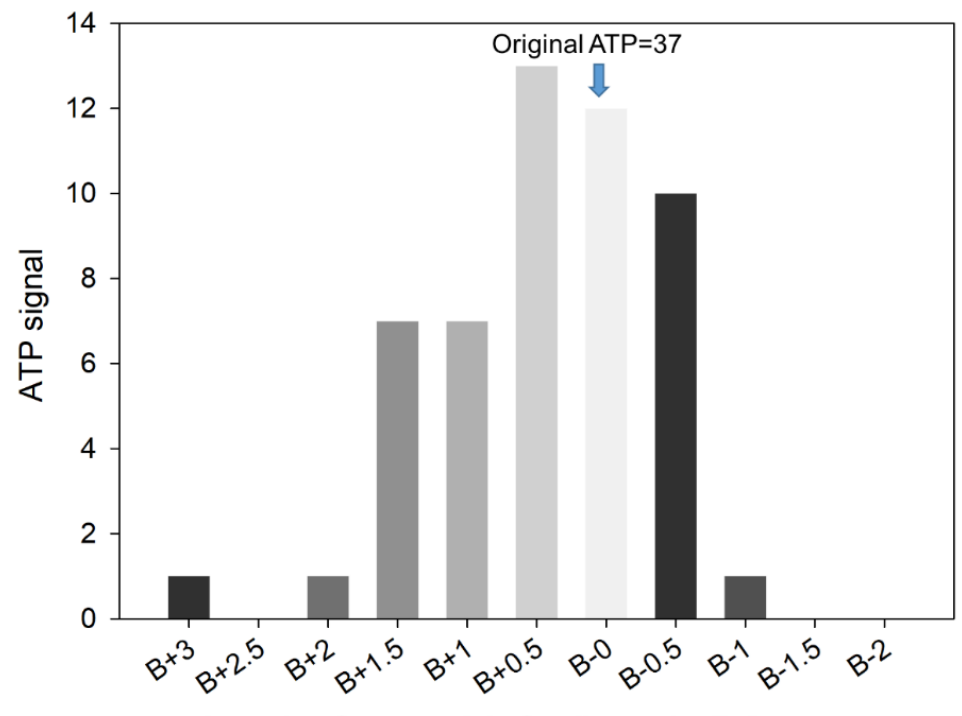

Location from particle feeding point B-0,cm

Fig. 3. Bacterial ATP detection results for the Beijing's PM iSCAPE'd as shown in Fig. 2. Numbers in the figure represent the distances $(\mathrm{cm})$ away from the sample feed point (B-0), and minus and plus signs represent locations toward negative and positive electrodes, respectively. Due to limited sample volume, $5 \mu \mathrm{L}$ was used for ATP measurement for each sampling point.

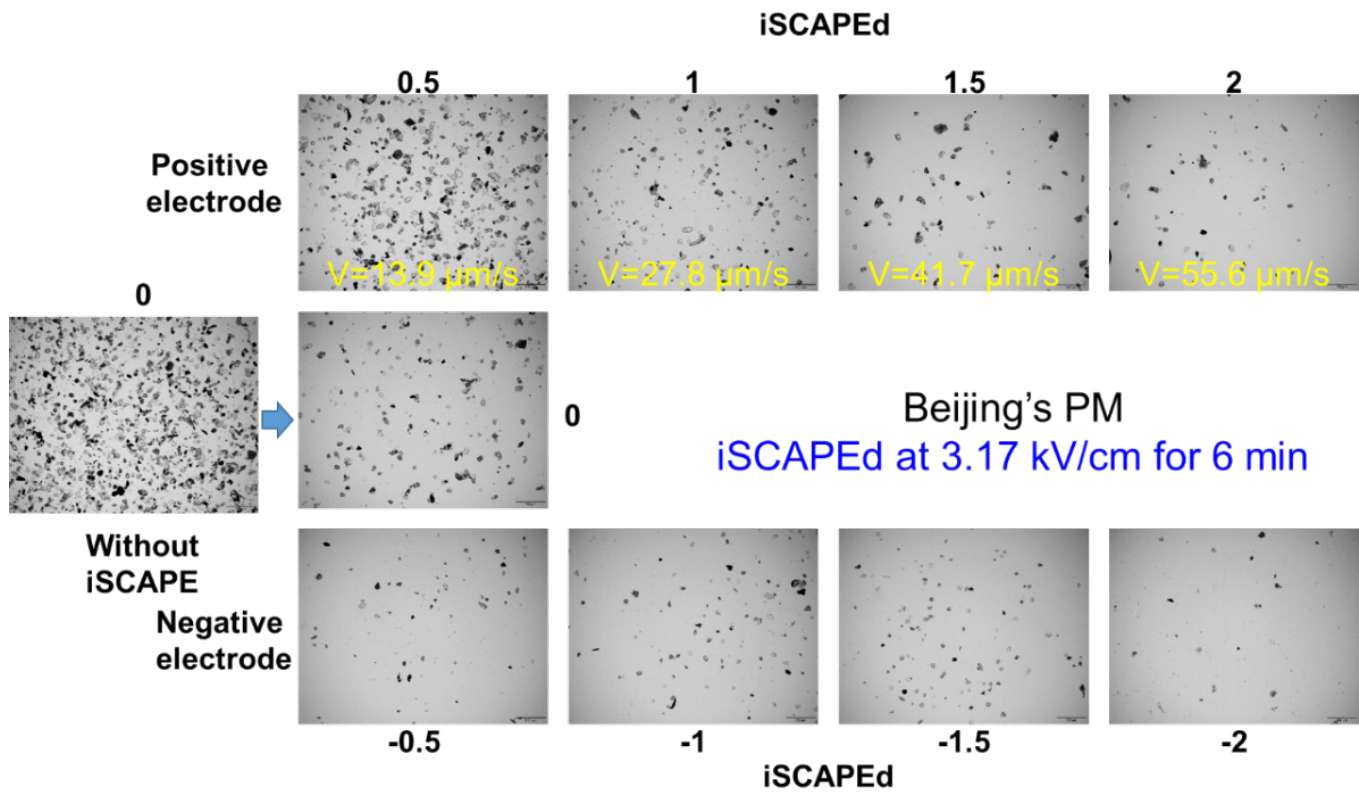

Fig. 4. Fine sieving of atmospheric particles from Beijing using the iSCAPE at $3.17 \mathrm{kV} \mathrm{cm}^{-1}$ for $6 \mathrm{~min}$. Videos of Beijing's PM iSCAPE'd for $3 \mathrm{~min}$ with particles along the particle moving line are further provided in File S1 (negative charges) and S2 (positive charges). For each sampling point, at least five images (200 $\mu \mathrm{m}$ scale bar) taken from different microscopic views of $10 \mu \mathrm{L}$ sample.

and road dust samples (Figs. S2, S3, and Fig. 5) at $3.17 \mathrm{kV} \mathrm{cm}^{-1}$ for $2 \mathrm{~min}$. As observed in Figs. S2 and S3 along with videos (File S3, S4, S5, and S6), the iSCAPE system was shown to efficiently sieve soil and road dust particles using their electrical mobility. Again, particles of different sources have demonstrated different mobility graphs under the same operating conditions (the electrostatic field strength and the same run time) given the same-viscosity mineral oil. To test high electrostatic field strength, a modified iSCAPE was used, e.g., shorter electrode distance $(3 \mathrm{~cm})$ but with the same voltage $(19 \mathrm{kV})$, and the results with Beijing soil sample are shown in 


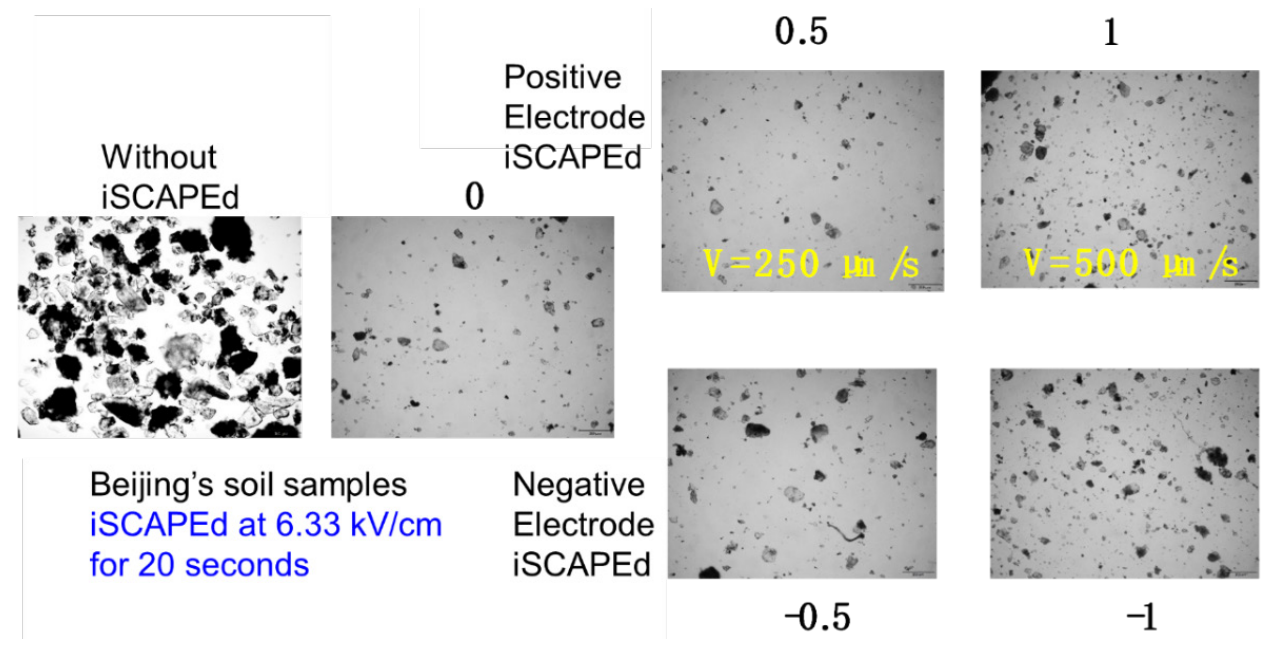

Fig. 5. Fine sieving of Beijing's soil samples using the iSCAPE at $6.33 \mathrm{kV} \mathrm{cm}^{-1}$ for 20 seconds. Videos of Beijing's soil sample iSCAPE'd are provided in File S7 (negative charges) and File S8 (positive charges). For each sampling point, at least five images (200 $\mu \mathrm{m}$ scale bar) taken from different microscopic views of $10 \mu \mathrm{L}$ sample.

Fig. 5. As observed from the figure, under higher electrostatic field strength $\left(6.33 \mathrm{kV} \mathrm{cm}^{-1}\right)$, all particles travelled much faster up to $500 \mu \mathrm{m} \mathrm{s}^{-1}$ for the location of $1 \mathrm{~cm}$ than the lower electrostatic field $\left(3.17 \mathrm{kV} \mathrm{cm}^{-1}\right)$, and even within 20 seconds the particles can be well sieved as seen in the figure. There was a clear contrast between samples before and after the iSCAPE test. Images of particles at other particle moving points on the line can be seen in File S7 and S8. In addition to air, these data showed that the iSCAPE system can be also applied to many other samples, and the particle sieving can be fine-controlled by adjusting the electrostatic field strength, particle charge and the run time. The particle electrical mobility per Eq. (1) is a function of particle charge, electrophoresis medium viscosity and particle diameter (Hinds, 1999). The bacterial particle charge can be attributed to two factors: ionizable groups $\left(\mathrm{NH}_{2}\right.$ and carboxyl $\left.(\mathrm{COOH})\right)$ or others present on the cell surface and the external particle frictions (Mainelis et al., 2001). To some extent, the latter can be modified by a manual charging process. Therefore, biological and non-biological particles with similar particle sizes could move differently under the same iSCAPE operating conditions.

The efficiency of the separation by the iSCAPE technology depends on the particle size distribution and particle charges. Here, we described the particle electrical mobility using Eq. (1) where $V_{d}$ and $E$ are known for particles at a particular location since the separation time is known. For particles carrying the same number of charges, the separation entirely depends on the particle size, i.e., the bigger particles move slowly, while smaller ones move faster. In the literature, differential mobility analyzer along with bipolar charging and condensation nucleus counter (CNC) is used to convert particle mobility distribution into particle size distribution directly for the nanoparticles (typically less than $0.1 \mu \mathrm{m}$ ) in the air by applying the Boltzmann's law modified by the Fuchs' charging theory with an extension to larger particles of geometric mean diameters of $1 \mu \mathrm{m}$ (Kousaka et al., 1985). In general, good agreements were observed between the conversion and actual measurements. However, the conversion can be influenced by the Brownian diffusion loss for particles of less than $15 \mathrm{~nm}$ and also the decreasing sheath air flow rate (Kousaka et al., 1985). In another relatively recent work, it was shown that Brownian dynamics (BD) simulation, which takes ion mobility and mass distributions into account, was a viable approach for the calculation of bipolar charge distributions (Gopalakrishnan et al., 2015). Also, they reported that the particle morphology had an influence on the bipolar charge distribution, but much weaker than the mobility-equivalent size. In this work, the particle mixture to be analyzed is complex, consisting of varying sizes of particles of different origins and materials. Of course the particle charge distribution is also unknown. Here, we intended to demonstrate an approach to separate particles with different electrical mobility from an already collected particle mixture. This technology opens up opportunities for many air pollution and its health effect experts to study collected 
ambient particles by size or their mobility attributes, thus adding additional capability to current available methods. In future efforts, similar to the literature, particle charging for the mixture needs to be externally modified in order to convert the particle mobility into particle size distribution or directly separate the particles from the mixture by size. An advantage from this effort is that once the particles can be separated solely by size, they can be used for many size-resolved post-analysis including their toxicity for an already collected ambient particle sample. This will significantly reduce the reliance on size-resolved ambient particle sampling, and make the size-resolved ambient particle analysis widely possible to academic communities and government agencies. Nonetheless, the iSCAPE system could be negatively impacted by the moistures in the sample and possible ions in the mineral oil. Additionally, for those non-charged particles they could remain at the sample feeding location without moving, and such a fraction needs to be determined for samples collected under different atmospheric conditions which play important roles in particle charging.

\section{CONCLUSIONS}

In this study, we invented a method named iSCAPE of enriching and finely separating particles, including bacteria, fungi, pollen, and viruses from a mixed atmospheric sample based on their electrical mobility. To examine particle health effect or haze formation, iSCAPE can use predetermined operating parameters to target specific particles in an air sample; additionally, it shows a potential in separating and purifying protein and chemical molecules in a biological sample. In fact, iSCAPE can theoretically extract any target from an environmental or medical sample, e.g., for improved PCR detection, by modifying the electrical field, mineral oil viscosity, run time, and particle charges. However, unlike conducting direct measurements of the air, charging the particles in a mixed sample is a complex process that requires further investigation.

Currently, we are confined to presenting quantitative results related to the electrical mobility of the particles. In the future, as suggested in the literature, externally charging the mixed sample or experimentally measuring the particle charges may facilitate the conversion of electrical mobility data into size distribution data. . Additionally, this could be also rapidly achieved using automatic microscopic particle recognition technique, which counts the numbers of $\mathrm{PM}_{2.5}$ and $\mathrm{PM}_{10}$ for any particular point along the separation line. Certainly, a large amount of work needs to be rapidly explored for the innovative applications of the invented iSCAPE in many different fields such as air pollution, clinical microbiology, and sample purification.

\section{ACKNOWLEDGMENTS}

This study was supported by the NSFC Distinguished Young Scholars Fund awarded to M. Yao (21725701), and National Natural Science Foundation of China (Grant Nos. 92043302, 22040101, 12041602). A patent has been submitted for the iSCAPE technology developed here prior to this manuscript submission. M. Yao conceived the research idea, and S. Xu and X. Li performed experiments with equal contributions.

\section{CONFLICT OF INTEREST}

The authors declare no conflict of interest.

\section{SUPPLEMENTARY MATERIAL}

Supplementary data associated with this article can be found in the online version at https://doi.org/10.4209/aaqr.200666

\section{REFERENCES}

Agranovski, V., Ristovski, Z., Hargreaves, M., Blackall, P.J., Morawska, L. (2003). Real-time 
measurement of bacterial aerosols with the UVAPS: Performance evaluation. J. Aerosol Sci. 34, 301-317. https://doi.org/10.1016/S0021-8502(02)00181-7

Alvarez, A.J., Buttner, M.P., Stetzenbach, L.D. (1995). PCR for bioaerosol monitoring: Sensitivity and environmental interference. Appl. Environ. Microbiol. 61, 3639-3644. https://doi.org/10. 1007/BF00871823

Forouzanfar, M.H., Alexander, L., Anderson, H.R., Bachman, V.F., Biryukov, S., Brauer, M. Burnett, R., Casey, D., Coates, M. M., Cohen, A., Delwiche, K., Estep, K., Frostad, J.J., Astha, K.C., Kyu, H.H., Moradi-Lakeh, M., Ng, M., Slepak, E.L., Thomas, B.A., Wagner, J., et al. (2013). Global, regional, and national comparative risk assessment of 79 behavioural, environmental and occupational, and metabolic risks or clusters of risks in 188 countries, 1990-2013: A systematic analysis for the global burden of disease study. Lancet 386, 2287-2323. https://doi.org/10.1016/S0140-6736(15)00128-2

Gopalakrishnan, R., McMurry, P.H., Hogan, C.J. (2015) The bipolar diffusion charging of nanoparticles: A review and development of approaches for non-spherical particles. Aerosol Sci. Technol. 49, 1181-1194. https://doi.org/10.1080/02786826.2015.1109053

Hewitt, G.W. (1957). The charging of small particles for electrostatic precipitation. Trans. Am. Inst. Electr. Eng. 76, 300-306. https://doi.org/10.1109/TCE.1957.6372672

Hinds, W.C. (1999). Aerosol technology: Properties, behavior, and measurement of airborne particles. John Wiley \& Sons.

Hospodsky, D., Yamamoto, N., Peccia, J. (2010). Accuracy, precision, and method detection limits of quantitative PCR for airborne bacteria and fungi. Appl. Environ. Microbiol. 76, 7004-7012. https://doi.org/10.1128/AEM.01240-10

Knutson, E.O., Whitby, K.T. (1975). Aerosol classification by electric mobility: Apparatus, theory, and applications. J. Aerosol Sci. 6, 443-451. https://doi.org/10.1016/0021-8502(75)90060-9

Kousaka, Y., Okuyama, K., Adachi, M. (1985). Determination of particle size distribution of ultrafine aerosols using a differential mobility analyzer. Aerosol Sci. Technol. 4, 209-225. https://doi.org/10.1080/02786828508959049

Li, J., Cao, J., Zhu, Y., Chen, Q., Shen, F., Wu, Y., Xu, S., Fan, H., Da, G., Huang, R.J., Wang, J., De Jesus, A.L., Morawska, L., Chan, C.K., Peccia, J., Yao, M. (2018). Global survey of antibiotic resistance genes in air. Environ. Sci. Technol. 52, 10975-10984. https://doi.org/10.1021/acs.est.8b02204

Li, J., Chen, H., Li, X., Wang, M., Zhang, X., Cao, J., Shen, F., Wu, Y., Xu, S., Fan, H., Da, G., Huang, R.J., Wang, J., Chan, C.K., De Jesus, A.L., Morawska, L., Yao, M. (2019). Differing toxicity of ambient particulate matter (PM) in global cities. Atmos. Environ. 212, 305-315. https://doi.org/10.101 6/j.atmosenv.2019.05.048

Lin, X., Reponen, T.A., Willeke, K., Grinshpun, S.A., Foarde, K.K., Ensor, D.S. (1999). Long-term sampling of airborne bacteria and fungi into a non-evaporating liquid. Atmos. Environ. 33, 4291-4298. https://doi.org/10.1016/S1352-2310(99)00169-7

Mainelis, G., Willeke, K., Baron, P., Reponen, T., Grinshpun, S.A., Gorny, R.L., Trakumas, S. (2001). Electrical charges on airborne microorganisms. J. Aerosol. Sci. 32, 1087-1110. https://doi.org/ 10.1016/S0021-8502(01)00039-8

Panyim, S., Roger, C. (1969). High resolution acrylamide gel electrophoresis of histones. Arch. Biochem. Biophys. 130, 337-346. https://doi.org/10.1016/0003-9861(69)90042-3

Sparkman, D.R. (1992). Paraffin wax as a vapor barrier for the PCR. Genome Res. 2, 180-181. https://doi.org/10.1101/gr.2.2.180

Ünlü, M., Morgan, M.E., Minden, J.S. (1997). Difference gel electrophoresis. A single gel method for detecting changes in protein extracts. Electrophoresis 18, 2071-2077. https://doi.org/10.1 002/elps.1150181133

Wei, K., Zou, Z., Yao, M. (2014). Charge levels and Gram ( \pm ) fractions of environmental bacterial aerosols. J. Aerosol Sci. 74, 52-62. https://doi.org/10.1016/j.jaerosci.2014.04.002

Xie, C., Shen, F., Yao, M. (2011). A novel method for measuring the charge distribution of airborne microbes. Aerobiologia 27, 135-145. https://doi.org/10.1007/s10453-010-9183-x

Xu, S., Yao, M. (2013). NanoPCR detection of bacterial aerosols. J. Aerosol Sci. 65, 1-9. https://doi.org/10.1016/j.jaerosci.2013.06.005

Yao, M., Mainelis, G. (2006a). Effect of physical and biological parameters on enumeration of bioaerosols by portable microbial impactors. J. Aerosol Sci. 37, 1467-1483. https://doi.org/10. 1016/j.jaerosci.2006.06.005 
Yao, M., Mainelis, G. (2006b). Utilization of natural electrical charges on airborne microorganisms for their collection by electrostatic means. J. Aerosol Sci. 37, 513-527. https://doi.org/10.101 6/j.jaerosci.2005.05.006

Yue, Y., Chen, H., Setyan, A., Elser, M., Dietrich, M., Li, J., Zhang, T., Zhang, X., Zheng, Y., Wang, J., Yao, M. (2018). Size-resolved endotoxin and oxidative potential of ambient particles in Beijing and Zürich. Environ. Sci. Technol. 52, 6816-6824. https://doi.org/10.1021/acs.est.8b01167

Zhang, T., Li, X., Wang, M., Chen, H., Yao, M. (2019). Microbial aerosol chemistry characteristics in highly polluted air. Sci. China Chem. 62, 1051-1063. https://doi.org/10.1007/s11426-0199488-3 